\title{
Magnetic behavior of curium dioxide with non-magnetic ground state
}

\author{
Fumiaki Niikura ${ }^{1}$ and Takashi Hotta ${ }^{1,2}$ \\ ${ }^{1}$ Department of Physics, Tokyo Metropolitan University, Hachioji, Tokyo 192-0397, Japan \\ ${ }^{2}$ Advanced Science Research Center, Japan Atomic Energy Agency, Tokai, Ibaraki 319-1195, Japan
}

(Dated: September 19, 2018)

\begin{abstract}
In order to understand magnetic behavior observed in $\mathrm{CmO}_{2}$ with non-magnetic ground state, we numerically evaluate magnetic susceptibility on the basis of a seven-orbital Anderson model with spin-orbit coupling. Naively we do not expect magnetic behavior in $\mathrm{CmO}_{2}$, since $\mathrm{Cm}$ is considered to be tetravalent ion with six $5 f$ electrons and the ground state is characterized by $J=0$, where $J$ is total angular momentum. However, there exists magnetic excited state and the excitation energy is smaller than the value of the Landé interval rule due to the effect of crystalline electric field potential. Then, we open a way to explain magnetic behavior in $\mathrm{CmO}_{2}$.

PACS numbers: 75.20.Hr, 75.40.Cx, 71.70.Ch, 71.27.+a
\end{abstract}

Curium is one of transuranium elements and it takes trivalent or tetravalent ion state. It is easy to understand that local $f$ electron number $n$ is equal to 6 and 7 for $\mathrm{Cm}^{4+}$ and $\mathrm{Cm}^{3+}$ ions, respectively. By following the well-known Hund's rules, we obtain that the ground-state multiplet for $\mathrm{Cm}^{3+}$ is characterized by $S=7 / 2$ and $L=0$, while for $\mathrm{Cm}^{4+}$, the multiplet is expressed by $S=3$ and $L=3$, where $S$ and $L$ denote spin and angular momenta, respectively. When we further include the effect of a spin-orbit interaction, the ground state for $\mathrm{Cm}^{3+}$ with $n=7$ is characterized by $J=S=7 / 2$, which is the same as that for $\mathrm{Gd}^{3+}$ ion. On the other hand, for the case of $\mathrm{Cm}^{4+}$ ion with $n=6$, the ground state is just the singlet with $J=0$. Thus, the magnetic behavior should be quite different between $\mathrm{Cm}^{3+}$ and $\mathrm{Cm}^{4+}$ ions.

Now we pay our attention to experimental results on $\mathrm{CmO}_{2}, \frac{1}{\text { w }}$ which are in a puzzling situation. When we naively deduce the valence of $\mathrm{Cm}$ ion from our experience in other actinide dioxides, Cm should be tetravalent, leading to non-magnetic ground state. However, from the neutron diffraction experiment, the effective moment $\mu_{\text {eff }} \sim 3.4 \mu_{\mathrm{B}}$ has been observed, where $\mu_{\mathrm{B}}$ is the Bohr magneton. In accordance with the large value of $\mu_{\mathrm{eff}}$, it has been also observed that the magnetic susceptibility follows the so-called Curie-Weiss behavior. At the first glance, these experimental results seem to contradict non-magnetic ground state of $\mathrm{Cm}^{4+}$ ion.

A possibility to resolve the contradiction is to consider the effect of $\mathrm{Cm}^{3+}$ magnetic impurity. However, the amount of $\mathrm{Cm}^{3+}$ deduced from the quantity of oxygen is apparently smaller than the value to explain the magnetic moment. Furthermore, there is no evidence in the neutron diffraction for the superlattice peak corresponding to a long-range rearrangement of oxygen sublattice to accommodate a mixture of $\mathrm{Cm}^{3+}$ and $\mathrm{Cm}^{4+}$ ions. From a theoretical viewpoint, it is an interesting issue to consider a mechanism to obtain magnetic behavior from the non-magnetic ground state.

One way to understand the problem is to consider seriously the magnetic excited state of $\mathrm{Cm}^{4+}$ ion, as already mentioned in the experimental paper $\stackrel{1}{=}$ Namely, when the magnetic excited state exists just above the non-magnetic ground state with a small excitation energy, it may be possible to understand magnetic behavior even for the non-magnetic ground state. This scenario seems to be quite simple, but we believe that it is worth to examine faithfully the scenario from a theoretical viewpoint.

In this paper, first we analyze the local $f$-electron state of $\mathrm{Cm}^{4+}$ ion on the basis of the Hamiltonian including Coulomb interactions, spin-orbit coupling $\lambda$, and crystalline electric field $(\mathrm{CEF})$ potential. If we ignore the CEF potential, the excitation energy $\Delta E$ is given by $\Delta E=\lambda / 6$ in an $L S$ coupling region, while it is expressed by $\Delta E=7 \lambda / 2+\eta$ in a $j-j$ coupling region, where $\eta$ is the energy shift depending on the CEF potential. When we further include the CEF potential, we find the relation of $\Delta E \propto \lambda^{4}$ in the $L S$ coupling region, which is smaller than the value in the Landé interval rule. Then, we evaluate the magnetic susceptibility on the basis of a seven-orbital Anderson model by exploiting a numerical renormalization group technique. It is found that the magnetic behavior actually appears for a realistic value of $\lambda$ even if we assume $\mathrm{Cm}^{4+}$ state.

Let us first discuss the local $f$-electron state. The model is given by ${ }^{2}$

$$
\begin{aligned}
& H_{\mathrm{f}}=\sum_{m, m^{\prime}} \sum_{\sigma, \sigma^{\prime}}\left(B_{m, m^{\prime}} \delta_{\sigma, \sigma^{\prime}}+\lambda \zeta_{m, \sigma, m^{\prime}, \sigma^{\prime}}\right) f_{m \sigma}^{\dagger} f_{m^{\prime} \sigma^{\prime}} \\
& +\sum_{m_{1} \sim m_{4}} \sum_{\sigma, \sigma^{\prime}} I_{m_{1} m_{2}, m_{3} m_{4}} f_{m_{1} \sigma}^{\dagger} f_{m_{2} \sigma^{\prime}}^{\dagger} f_{m_{3} \sigma^{\prime}} f_{m_{4} \sigma},
\end{aligned}
$$

where $B_{m, m^{\prime}}$ is the CEF potential, $\sigma=+1(-1)$ for up (down) spin, $f_{m \sigma}$ is the annihilation operator for $f$ electron with spin $\sigma$ and $z$-component $m$ of angular momentum $\ell=3, \delta_{\sigma \sigma^{\prime}}$ is the Kronecker's delta, $\lambda$ is the spinorbit coupling, $\zeta_{m, \pm 1, m, \pm 1}= \pm m / 2, \zeta_{m \pm 1, \mp 1, m, \pm 1}=$ $\sqrt{12-m(m \pm 1)} / 2$, and zero for the other cases. The Coulomb integral $I$ is expressed by the combination of Slater-Condon parameters such as $F^{0}, F^{2}, F^{4}$, and $F^{6} \underline{\underline{3}}$

Here we briefly explain the parameters of the local Hamiltonian $H_{\mathrm{f}}$. Concerning Slater-Condon parameters, first we set $F^{0}=10 \mathrm{eV}$ by hand, since we are not interested in the determination of the absolute value of the ground state energy. Others are determined so as 


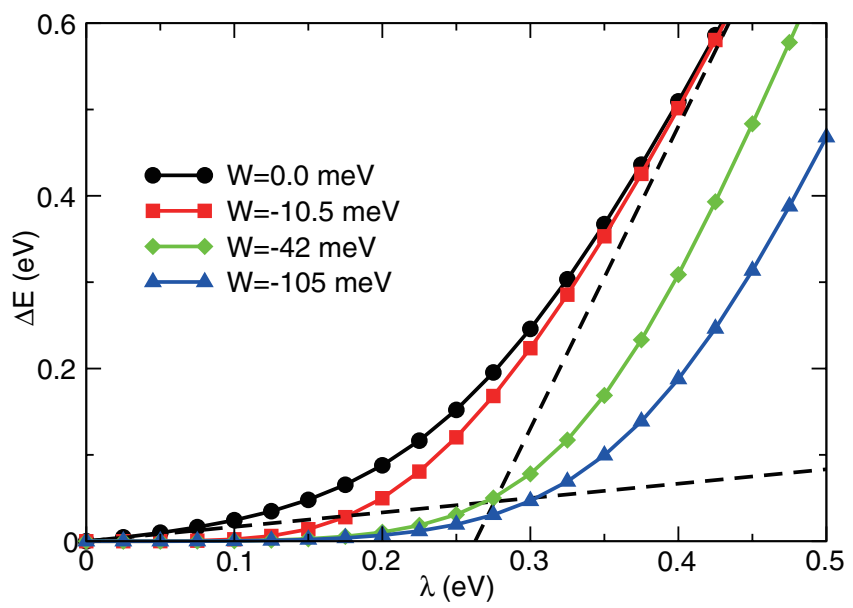

FIG. 1: Excitation energy $\Delta E$ vs. spin-orbit coupling $\lambda$ for $n=6$. Thick solid curves denote the calculated results with different values of the CEF potential $W$. Broken lines indicate the two limiting situations as $\Delta E=\lambda / 6$ for small $\lambda$ and $\Delta E=7 \lambda / 2+\eta$ for large $\lambda$, where $\eta$ is the energy shift depending on the CEF potential.

to reproduce excitation spectra of $\mathrm{U}^{4+}$ ion with two $5 f$ electrons .4 Here we show only the results: $F^{2}=6.36 \mathrm{eV}$, $F^{4}=5.63 \mathrm{eV}$, and $F^{6}=4.13 \mathrm{eV}$. As for spin-orbit coupling $\lambda$, it may be possible to use an experimental value, but in this paper, we change the value of $\lambda$ as a parameter to control the situation from the $L S$ coupling to $j$ - $j$ coupling schemes. Since the fluorite structure belongs to $O_{\mathrm{h}}$ point group, $B_{m, m^{\prime}}$ is given by using a couple of CEF parameters, $B_{4}^{0}$ and $B_{6}^{0}$, which are traditionally expressed as $B_{4}^{0}=W x / 15$ and $B_{6}^{0}=W(1-|x|) / 180$, respectively ${ }^{5,6}$ Here $W$ determines the energy scale of the CEF potential, while $x$ specifies the CEF scheme.

Now we summarize the experimental facts on the CEF energy levels of actinide dioxides: For $\mathrm{UO}_{2}$, the ground state is $\Gamma_{5}^{+}$triplet and the first excited state is $\Gamma_{3}^{+}$doublet with the excitation energy $150 \mathrm{meV} \stackrel{\underline{7}}{ }$ For $\mathrm{NpO}_{2}$, the ground and first excited states are, respectively, $\Gamma_{8}^{-(2)}$ and $\Gamma_{8}^{-(1)}$ quartets with the excitation energy $55 \mathrm{meV}$ 로 For $\mathrm{PuO}_{2}$, the ground state is $\Gamma_{1}^{+}$singlet, while the first excited state is $\Gamma_{4}^{+}$triplet with the excitation energy $123 \mathrm{meV} \underline{\underline{9} .10}$ In order to reproduce totally the above CEF level schemes, we have chosen $W=-10.5 \mathrm{meV}$ and $x=0.62$ in the discussion of the ground state of $\mathrm{AmO}_{2}, \frac{11}{\lambda}$

In Fig. 1, we show the excitation energy $\Delta E$ vs. $\lambda$ for various values of $W$. Note that at $\lambda=0$, the ground state is highly degenerate. Here we set $\Delta E=0$ at $\lambda=0$ in order to visualize how the degeneracy is lifted by the inclusion of $\lambda$. First we consider the case of $W=0$, in which the ground state is characterized by $J=0$, while the first excited state is given by $J=1$. In this case, we obtain $\Delta E=\lambda / 6$ in the $L S$ coupling region from the so-called Landé interval rule. On the other hand, in the $j$ - $j$ coupling region, the ground state is expressed by the closed shell of $j=5 / 2$ sextet and the exited state is given by the one-electron excitation from $j=5 / 2$ sextet to $j=7 / 2$ octet, leading to the $J=1$ excited state. Thus, we obtain $\Delta E=7 \lambda / 2+\eta$ in the $j$ - $j$ coupling scheme, where $\eta$ denotes the energy shift depending on the CEF potentials.

Now we include the effect of the CEF potential. As shown in Fig. 1, the excitation energy becomes small when we set $W=-10.5 \mathrm{meV}$, which has been determined so as to reproduce the CEF energy levels of other actinide dioxides. Both the ground state and the first excited state energies are decreased by the CEF potential, but the reduction of the first excited state energy is large in comparison with that of the ground state energy. From the analysis of our numerical results, we find the relation of $\Delta E \propto \lambda^{4}$, suggesting that $\Delta E$ is suppressed for small $\lambda$ in comparison with the relation of $\Delta E=\lambda / 6$ for $W=0$. When we further increase the magnitude of $W$, the excitation energy is getting smaller and smaller, as observed in Fig. 1. Thus, it is concluded that the excitation energy of the $f^{6}$ electron system is smaller than we have naively expected due to the combined effect of Coulomb interactions, spin-orbit interaction, and the CEF potential.

Let us now include the hybridization between localized and conduction electrons. The Hamiltonian is the sevenorbital Anderson model, $\stackrel{12}{\rightleftharpoons}$ given by

$$
H=\sum_{\mathbf{k}, \sigma} \varepsilon_{\mathbf{k}} c_{\mathbf{k} \sigma}^{\dagger} c_{\mathbf{k} \sigma}+\sum_{\mathbf{k}, \sigma, m}\left(V_{m} c_{\mathbf{k} \sigma}^{\dagger} f_{m \sigma}+\text { h.c. }\right)+H_{\mathrm{f}}
$$

where $\varepsilon_{\mathbf{k}}$ denotes conduction electron dispersion, $c_{\mathbf{k} \sigma}$ indicates the annihilation operator for conduction electron with momentum $\mathbf{k}$ and spin $\sigma$, and $V_{m}$ is the hybridization between conduction and $f$ electrons.

Note that we consider only $a_{\mathrm{u}}$ single conduction band with xyz symmetry composed of oxygen $2 p$ electrons. Since oxygen ions surrounding actinide ions are located in the $[1,1,1]$ direction, there should exist a conduction band composed of $2 p$ electrons with xyz symmetry. This picture seems to be consistent with band-structure calculation,,$\frac{13}{=}$ but we assume the ignorance of $t_{1 \mathrm{u}}$ and $t_{2 \mathrm{u}}$ bands. Here we note that the hybridization occurs between the states with the same symmetry of local $f$ electron state. Since the $a_{\mathrm{u}}$ conduction band has xyz symmetry, we set $V_{2}=-V_{-2}=V$ and zero for other $m$, where $V$ is fixed as $V=0.05 \mathrm{eV}$. In order to adjust the local $f$-electron number $n$, we appropriately change the chemical potential in the actual calculation, although we do not explicitly show such a term. A half of the bandwidth of $a_{\mathrm{u}}$ conduction band is set as $1 \mathrm{eV}$, which is the energy unit in the following calculations.

In this paper, we analyze the seven-orbital Anderson model by using the numerical renormalization group (NRG) technique $\stackrel{14,15}{1 n}$ In NRG calculations, in order to consider efficiently the conduction electrons near the Fermi energy, the momentum space is logarithmically discretized and the conduction electron states are characterized by "shell" labeled by $N$. The shell of $N=0$ denotes an impurity site including $f$ electrons. The Hamiltonian 


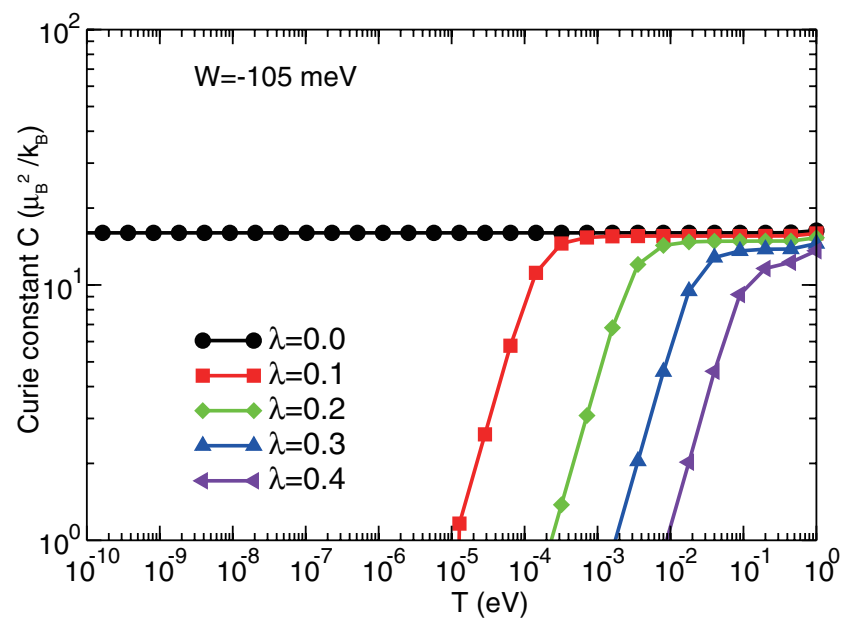

FIG. 2: Curie constant $C$ vs. temperature $T$ for various values of $\lambda$.

is transformed into the recursion form as

$$
H_{N+1}=\sqrt{\Lambda} H_{N}+t_{N} \sum_{\sigma}\left(c_{N \sigma}^{\dagger} c_{N+1 \sigma}+c_{N+1 \sigma}^{\dagger} c_{N \sigma}\right)
$$

where $\Lambda$ is a parameter for logarithmic discretization, $c_{N \sigma}$ denotes the annihilation operator of conduction electron in the $N$-shell, and $t_{N}$ is "hopping" of electron between $N$ - and $(N+1)$-shells, given by

$$
t_{N}=\frac{\left(1+\Lambda^{-1}\right)\left(1-\Lambda^{-N-1}\right)}{2 \sqrt{\left(1-\Lambda^{-2 N-1}\right)\left(1-\Lambda^{-2 N-3}\right)}} .
$$

The initial term $H_{0}$ is given by

$$
H_{0}=\Lambda^{-1 / 2}\left[H_{\mathrm{f}}+\sum_{m \sigma} V_{m}\left(c_{0 \sigma}^{\dagger} f_{m \sigma}+f_{m \sigma}^{\dagger} c_{0 \sigma}\right)\right] .
$$

In this paper, $\Lambda$ is set as 5 and we keep 4500 low-energy states for each renormalization step.

In order to see magnetic properties, we evaluate the magnetic susceptibility of $f$-electron, defined by

$$
\chi=\frac{1}{T} \lim _{N \rightarrow \infty}\left[\frac{\operatorname{Tr} M_{z, N}^{2} e^{-H_{N} / T}}{\operatorname{Tr} e^{-H_{N} / T}}-\frac{\operatorname{Tr} S_{z, N}^{2} e^{-H_{N}^{0} / T}}{\operatorname{Tr} e^{-H_{N}^{0} / T}}\right],
$$

where $T$ denotes a logarithmic temperature given by $T=\Lambda^{-(N-1) / 2}$ in the NRG calculation, $H_{N}^{0}$ is the Hamiltonian without $H_{\mathrm{f}}$, and a magnetic moment along the $z$-axis is defined by

$$
M_{z, N}=\mu_{\mathrm{B}} \sum_{m, \sigma}\left(m+g_{s} \sigma / 2\right) f_{m \sigma}^{\dagger} f_{m \sigma}+S_{z, N}
$$

Here $\mu_{\mathrm{B}}$ is the Bohr magneton, $g_{s}$ is electron $g$-factor given by $g_{s}=2$, and

$$
S_{z, N}=g_{s} \mu_{\mathrm{B}} \sum_{n=0}^{N} \sum_{\sigma}(\sigma / 2) c_{n \sigma}^{\dagger} c_{n \sigma} .
$$

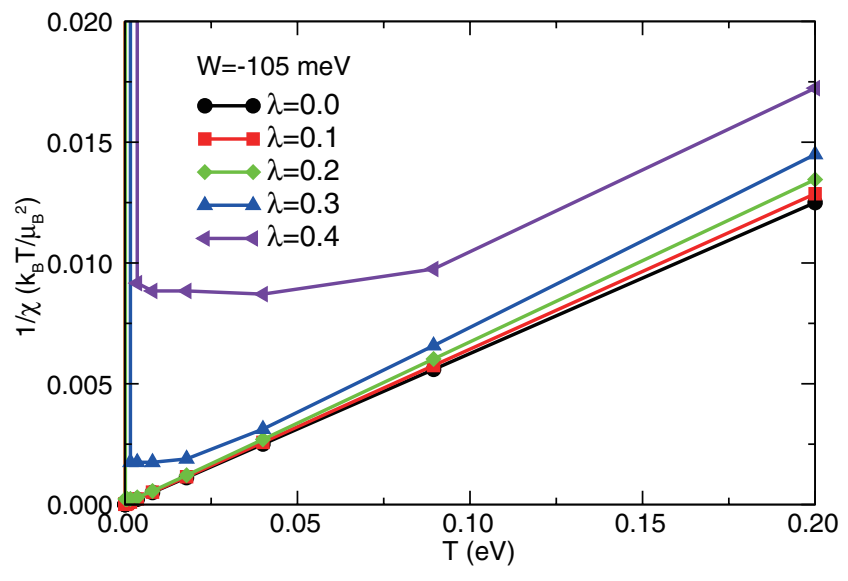

FIG. 3: Inverse susceptibility $1 / \chi$ vs. temperature $T$ for various values of $\lambda$. The CEF potential is set as $W=-105 \mathrm{meV}$.

Note that we consider only the susceptibility for the $z$ component of the magnetic moment, but we have performed the calculations for all directions of the magnetic moment. Then, we have checked that the susceptibilities for the magnetic moments along $x$-, $y$-, and $z$-directions take the same values due to the cubic symmetry. In the following, we show only the results for the susceptibility for the magnetic moment along the $z$-axis.

In Fig. 2, we show the Curie constant $C$ as a function of $T$. In NRG calculations, it is possible to evaluate precisely the Curie constant $C$ by using the relation $T \chi$. We note that the finite value of $C$ suggests the emergence of of magnetic behavior even for the non-magnetic ground state. Here the CEF potential is chosen as $W=-105 \mathrm{meV}$ in order to obtain the small excitation energy. Concerning the large magnitude of $W$, we will provide a brief comment later.

First let us consider the case of $\lambda=0$, in which we find $C=16 \mu_{\mathrm{B}}^{2} / k_{\mathrm{B}}$ almost irrespective of a temperature, as observed in Fig. 2. Note that the residual entropy from the localized magnetic moment is due to the speciality of the present model in which we consider only the hybridization with single $a_{\mathrm{u}}$ band. If we further include $t_{1 \mathrm{u}}$ and $t_{2 \mathrm{u}}$ bands, the residual entropy will be released due to the Kondo effect.

Now we consider the meaning of " 16 " in the value of $C$ for $\lambda=0$. In this situation, the ground state is characterized by $S=3$ and $L=3$ due to the Hund's rules, indicating 49-fold degeneracy in the ground state. When we include the effect of CEF potential, we note that the CEF potentials act on the charge, not on the spin. Namely, the septet of $L=3$ splits into one single and two triplets due to the effect of the cubic CEF potentials. For $W<0$ and $0<x<1$, the lowest energy state is found to be the singlet. Thus, the ground state becomes septet characterized by $S=3$. As is well known, the Curie constant of the susceptibility of the magnetic moment $M=L+g_{s} S=g_{J} J$ is given by $C=g_{J}^{2} \mu_{\mathrm{B}}^{2} J(J+1) /\left(3 k_{\mathrm{B}}\right)$, where $g_{J}$ is the Landé's $g$-factor. In the present case, 
$J$ is composed only of electron spins and thus, we set $J=S=3$ and $g_{J}=g_{s}=2$, leading to $C=16 \mu_{\mathrm{B}}^{2} /\left(3 k_{\mathrm{B}}\right)$. Such a large value of $C$ at $\lambda=0$ is related to the appearance of magnetism for $\lambda>0$.

When we increase the value of $\lambda, C$ should be decreased, since the ground state becomes the singlet characterized by $J=0$. However, as shown in Fig. 2, the Curie constant does not vanish immediately, when we increase the value of $\lambda$. In fact, as observed in the curve for $\lambda=0.1, C$ is still constant in the temperature region of $T \gtrsim 10^{-4}$ due to the smallness of the excitation energy. When $\lambda$ is further increased, the temperature dependence of the Curie constant becomes significant. For $\lambda=0.4, C$ becomes small drastically at a temperature as large as $T \sim 10^{-2}$.

In order to compare the numerical results with the experimental ones, we plot the inverse susceptibility $1 / \chi$ in Fig. 3. Here we use a large value for CEF potential as $W=-105 \mathrm{meV}$, in order to reproduce the small excitation energy. We note that $1 / \chi$ is shown in the linear scale of the temperature, even though the low-temperature precise behavior in the logarithmic-scale calculation cannot be observed in the linear scale for the temperature. Note also that except for $\lambda=0,1 / \chi$ diverges near $T \sim 0$, since the ground state is singlet and the Curie constant eventually goes to zero at low temperatures. In the expression of $1 / \chi$ in the linear scale, even for $\lambda=0.3$, we find the region of Curie-Weiss-like behavior in a temperature region of $T \gtrsim 0.01$. For $\lambda=0.4$, it is difficult to observe the Curie-Weiss-like behavior in the temperature range of $T<0.1$. From these results, we conclude that the magnetic behavior of $\mathrm{CmO}_{2}$ can be explained even for the non-magnetic ground state of $\mathrm{Cm}^{4+}$ if we appropriately choose the parameters in the model.

In the present scenario, the magnetic behavior should disappear at low enough temperatures. However, it will be difficult to perform low-temperature experiments for $\mathrm{CmO}_{2}$. An executable test is to measure the temperature dependence of the magnetic moment in the neutron diffraction experiment. If the present scenario is correct, it is possible to detect the reduction of the moment due to the decrease of temperature.

Finally, we briefly comment on the value of $W$. In the present calculation for $\chi$, the value of $W$ may be too large in comparison with that for other actinide dioxides. 11 Namely, our result indicates that such a large CEF potential is required to explain the magnetic behavior in $\mathrm{CmO}_{2}$ on the basis of the model calculations. A possible detection of the large CEF effect in $\mathrm{CmO}_{2}$ will be another test for the present scenario.

In summary, we have analyzed the magnetic susceptibility of $\mathrm{CmO}_{2}$ on the basis of the seven-orbital Anderson model by using the NRG technique. We have actually found that the magnetic behavior appears even if the ground state of $\mathrm{Cm}$ ion is non-magnetic, since the excitation energy between ground and magnetic excited states becomes small due to the combined effect of Coulomb interactions, spin-orbit coupling, and CEF potential. We believe that the scenario works to understand the magnetism in $\mathrm{CmO}_{2}$.

The authors thank S. Kambe and Y. Tokunaga for discussions on actinide dioxides. This work has been supported by a Grant-in-Aid for for Scientific Research on Innovative Areas "Heavy Electrons" (No. 20102008) of The Ministry of Education, Culture, Sports, Science, and Technology, Japan. The computation in this work has been done using the facilities of the Supercomputer Center of Institute for Solid State Physics, University of Tokyo.
1 L. R. Morss, J. W. Richardson, Jr., C. W. Williams, G. H. Lander, A. C. Lawson, N. M. Edelstein, and G. V. Shalimoff, J. Less-Common Metals 156, 273 (1989).

${ }^{2}$ In this paper, we use such units as $\hbar=k_{\mathrm{B}}=1$.

3 See, for instance, J. C. Slater, Quantum Theory of Atomic Structure, (McGraw-Hill, New York, 1960).

4 E. Eliav, U. Kaldor, and Y. Ishikawa, Phys. Rev. A 51, 225 (1995).

${ }^{5}$ K. R. Lea, M. J. M. Leask, and W. P. Wolf, J. Phys. Chem. Solids 23, 1381 (1962).

6 M. T. Hutchings, Solid State Phys. 16, 227 (1964).

7 G. Amoretti, A. Blaise, R. Caciuffo, J. M. Fournier, M. T. Hutchings, R. Osborn, and A. D. Taylor, Phys. Rev. B 40, 1856 (1989).

8 J. M. Fournier, A. Blaise, G. Amoretti, R. Caciuffo, J.
Larroque, M. T. Hutchings, R. Osborn, and A. D. Taylor, Phys. Rev. B 43, 1142 (1991).

9 S. Kern, C.-K. Loong, G. L. Goodman, B. Cort, and G. H. Lander, J. Phys.: Condens. Matter 2, 1933 (1990).

10 S. Kern, R. A. Robinson, H. Nakotte, G. H. Lander, B. Cort, P. Watson, and F. A. Vigil, Phys. Rev. B 59, 104 (1999).

11 T. Hotta, Phys. Rev. B 80, 024408 (2009).

12 T. Hotta, J. Phys. Soc. Jpn. 74, 1275 (2005).

13 T. Maehira and T. Hotta, J. Magn. Magn. Mater. 310, 754 (2007).

14 K. G. Wilson, Rev. Mod. Phys. 47, 773 (1975).

15 H. R. Krishna-murthy, J. W. Wilkins, and K. G. Wilson, Phys. Rev. B 21, 1003 (1980). 